\title{
Laboratory Diagnostic Methods for Clostridioides difficile Infection: the First Systematic Review and Meta-analysis in Korea
}

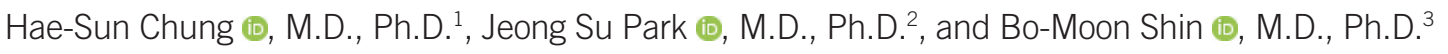 \\ ${ }^{1}$ Department of Laboratory Medicine, Ewha Womans University College of Medicine, Seoul, Korea; ${ }^{2}$ Department of Laboratory Medicine, Seoul National \\ University Bundang Hospital, Seongnam, Korea; ${ }^{3}$ Department of Laboratory Medicine, Sanggye Paik Hospital, School of Medicine, Inje University, Seoul, \\ Korea
}

Background: Various methods are used for the diagnosis of Clostridioides difficile infection (CDI). We systematically analyzed and investigated the performance of current laboratory diagnostic methods for CDI.

Methods: We performed systematic review and meta-analysis of studies in PubMed, Web of Science, Cochrane Library, and KoreaMed. The following methods were evaluated: glutamate dehydrogenase (GDH) enzyme immunoassays (GDH EIAs), toxin A and B detection by enzyme immunoassays (toxin $A B$ EIAs), and nucleic acid amplification tests (NAATs) for $C$. difficile toxin genes. The sensitivity, specificity, positive predictive value (PPV), and negative predictive value (NPV) of each method were calculated.

Results: Based on 39 studies, the pooled sensitivities/specificities were $92.7 \% / 94.6 \%$, $57.9 \% / 97.0 \%$, and $90.0 \% / 95.8 \%$ for GDH EIAs, toxin AB EIAs, and NAATs, respectively, compared with those of toxigenic culture. The pooled sensitivities of automated ElAs were significantly higher than those of non-automated EIAs for both GDH and toxins A and B. The pooled sensitivity of Xpert $C$. difficile was significantly higher than those of other NAATs. PPVs increased as CDI prevalence increased, and NPVs were excellent when CDI prevalence was low; at CDI prevalence of $5 \%, P P V=37 \%-65 \%$ and NPV $=97 \%-100 \%$; at CDI prevalence of 50\%, PPV $=92 \%-97 \%$ and NPV $=65 \%-98 \%$.

Conclusions: Toxin AB EIAs still show unsatisfactory sensitivity, whereas GDH EIAs and NAATs show relatively high sensitivity. However, toxin AB EIAs are the most specific tests. This study may provide useful information for $\mathrm{CDI}$ diagnosis.

Key Words: Clostridioides difficile, Clostridioides difficile infection, Laboratory diagnostic methods, Systematic review, Meta-analysis

\author{
Received: February 28, 2020 \\ Revision received: April 2, 2020 \\ Accepted: September 8, 2020 \\ Corresponding author: \\ Bo-Moon Shin, M.D., Ph.D. \\ Department of Laboratory Medicine, \\ Sanggye Paik Hospital, School of Medicine, \\ Inje University, 1342 Dongil-ro, Nowon-gu, \\ Seoul 01757, Korea \\ Tel: +82-2-950-1227 \\ Fax: +82-2-950-1244 \\ E-mail: bmshin@unitel.co.kr
}

\section{INTRODUCTION}

Clostridioides difficile (formerly known as Clostridium difficile) infection (CDI) is one of the most common healthcare-associated infections. $C$. difficile is an anaerobic gram-positive, sporeforming, toxin-producing bacillus that is transmitted among humans through the fecal-oral route, as a result of ingestion of spores. Colonization of $C$. difficile is prevented by barrier proper- ties of the fecal microbiota; weakening of this resistance by antibiotics is a major risk factor for disease. Toxin production is the key to pathogenesis, which leads to colonocyte death, loss of intestinal barrier function, and neutrophilic colitis. CDI can cause a variety of clinical manifestations, including asymptomatic colonization, mild diarrhea, toxic megacolon, and death [1, 2].

Various methods are used to diagnose CDI [1, 2], including detection of glutamate dehydrogenase (GDH) —an antigen se- 
creted by $C$. difficile - through enzyme immunoassays (GDH $E(A s)$, detection of toxins $A$ or $B$ of $C$. difficile strains through enzyme immunoassays (toxin $A B$ EIAs), or nucleic acid amplification tests (NAATs) for $C$. difficile toxin genes. Each assay has advantages and disadvantages and exhibits performance differences. When toxin production cannot be confirmed, GDH EIAs may be used to determine the presence of $C$. difficile. GDH EIAs are convenient and inexpensive tests with a rapid turnaround time and can be used as screening tests. GDH ElAs are very sensitive but not specific, because GDH is present in both toxigenic and nontoxigenic strains of $C$. difficile and therefore, to confirm the presence of a toxigenic strain, should be accompanied by an additional test, such as a toxin $A B$ EIA or NAAT. Toxin $A B$ ElAs are also rapid, inexpensive, and easy-to-perform tests that were widely adopted by many laboratories. Toxin $A B$ EIAs have high specificity but unacceptably low sensitivity and are no longer recommended as stand-alone tests for CDI diagnosis $[1,2]$.

Many commercialized NAATs, including PCR of toxin A and B genes, have been used. Most assays detect the toxin $B$ gene alone or both toxin $A$ and toxin $B$ genes. A multiplex PCR test that detects genes of multiple pathogens that cause diarrhea, including $C$. difficile toxin genes, is also available. NAATs are rapid tests with high specificity and sensitivity for $C$. difficile detection. However, NAATs can detect clinically insignificant infections such as asymptomatic carriage. Additionally, NAATs require trained personnel and are associated with high costs for clinical application. The use of multiple assays for CDI diagnosis suggests that no single test is optimal. In fact, several guidelines recommend combining test methods or using a multi-step algorithm $[3,4]$.

We systematically analyzed the current laboratory diagnostic methods for CDI. A literature review and meta-analysis were performed to investigate the usefulness of the diagnostic methods by calculating the sensitivity, specificity, positive predictive value (PPV), and negative predictive value (NPV) of each method. This study is an update of the previous meta-analysis [3] and analyzed the latest Korean data. In addition, it represents the first systematic review and meta-analysis of laboratory diagnostic methods for CDI in Korea.

\section{MATERIALS AND METHODS}

\section{Search strategy}

In 2016, the European Society of Clinical Microbiology and Infectious Diseases (ESCMID) study group published CDI diag- nostic methods evaluation based on studies evaluating CDI diagnostic methods published between January 2009 and June 2014 [3]. We applied the same search criteria as those used in that report. We searched PubMed, Web of Science, and Cochrane Library for studies written in English language and published from June 2014 to July 2018, and KoreaMed for Korean studies published from January 2009 to July 2018, with an additional criterion that the study should have been conducted in Korea.

\section{Index test}

We reviewed data from three diagnostic methods commonly used in clinical laboratories: GDH EIAs, toxin AB EIAs, and NAATs. EIAs are available in a well-type format (results are displayed as a color change that can be detected visually or spectrophotometrically) or a membrane-type format (results can be visually read from a membrane). Some EIAs are performed through an automated process that minimizes the manual process during the test. Among NAATs, Xpert $C$. difficile (Cepheid, Sunnyvale, CA, USA), BD Max Cdiff (Becton Dickinson, Sparks, MD, USA), and AdvanSure CD (LG Chem., Seoul, Korea) have been used with a relatively high frequency in Korea [5]. The information for each diagnostic method is shown in Table 1.

\section{Reference test}

Reference tests against which index tests were compared were toxigenic culture (TC) and the cell cytotoxicity neutralization assay (CCNA). Except for studies that used $C$. difficile culture, TC was used as a reference test in Korean studies. C. difficile culture was accepted as a reference test for comparison with GDH assays.

\section{Eligibility criteria and selection process}

Studies eligible for inclusion had to: (1) describe original research, (2) compare a commercially available index test with a reference test (CCNA or TC), (3) perform the tests on C. difficilenegative and-positive clinical human stool samples and (4) provide sufficient information to recalculate sensitivity and specificity and their confidence intervals (Cls).

Studies were excluded if: (1) the reference test was not clearly stated, (2) the reference test was performed only on positive, negative, or discordant samples (to exclude partial verification bias), (3) not all samples were tested by the same reference test, (4) the reference method was a composite of more than one test, (5) the reference method included clinical data for interpretation, (6) the index test was partly used as reference test, (7) the manufacturers' instructions were not followed for index 
Chung HS, et al.

Systematic review of laboratory diagnosis of CDI

Table 1. Pooled sensitivities and specificities of index tests

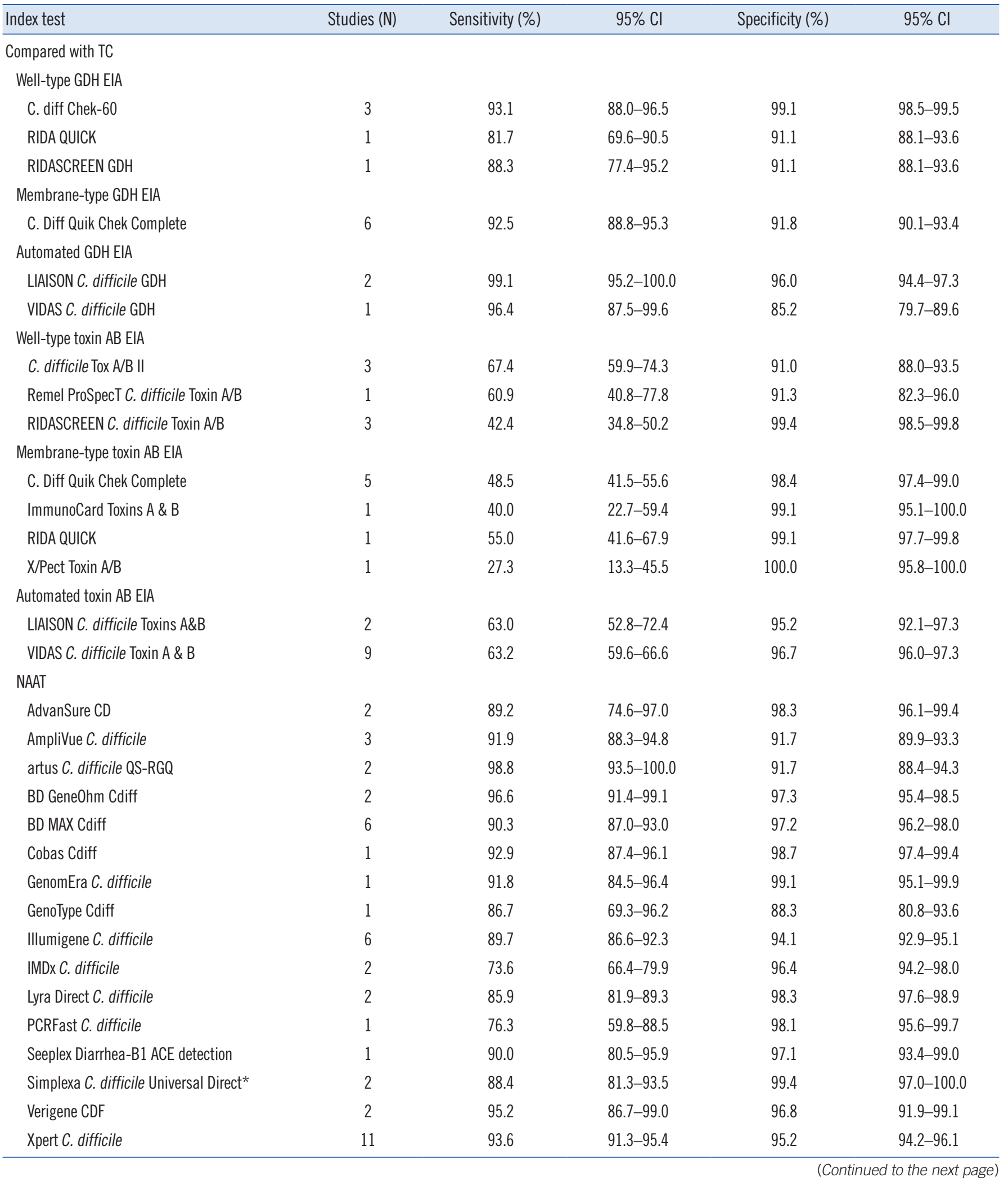


Table 1. Continued

\begin{tabular}{lccccc}
\hline Index test & Studies (N) & Sensitivity (\%) & $95 \% \mathrm{Cl}$ & Specificity (\%) & $95 \% \mathrm{Cl}$ \\
\hline $\begin{array}{l}\text { Compared with C. difficile culture } \\
\text { Well-type GDH EIA }\end{array}$ & & & & & \\
$\quad$ C. diff Chek-60 & 1 & 88.1 & $73.6-95.5$ & 99.6 & $97.5-100.0$ \\
$\quad$ RIDASCREEN GDH & 1 & 87.8 & $78.2-94.3$ & 93.7 & $90.9-95.8$ \\
$\quad$ Membrane-type GDH EIA & & & & & \\
$\quad$ C. Diff Quik Chek Complete & 1 & 94.1 & $85.6-98.3$ & 94.6 & $91.7-96.7$ \\
$\quad$ C. Diff Quik Chek-60 & 2 & 94.3 & $92.3-95.9$ & 96.0 & $95.3-96.6$ \\
$\quad$ Automated GDH EIA & & & & & \\
$\quad$ LIAISON C. difficile GDH & 1 & 100.0 & $89.6-100.0$ & 96.5 & $93.3-98.3$ \\
$\quad$ VIDAS C. difficile GDH & 4 & 95.4 & $93.9-96.7$ & 90.9 & $89.9-91.7$ \\
\hline
\end{tabular}

*Multiplex PCR test that detects the genes of multiple pathogens that cause multiple diarrhea, including $C$. difficile toxin genes.

Abbreviations: $\mathrm{Cl}$, confidence interval; TC, toxigenic culture; GDH, glutamate dehydrogenase; EIA, enzyme immunoassay; NAAT, nucleic acid amplification test.

testing or sample collection, (8) only selected samples were included, (9) insufficient information was provided, (10) clinical human stool samples were not included, (11) no commercial diagnostic test was investigated, or (12) only culture methods were evaluated.

Study eligibility was assessed in a two-step selection process by two independent investigators. Initially, one author identified the potentially relevant studies by screening titles and abstracts, and then two authors evaluated their eligibility for being included in this study through a full text assessment. Inconsistencies were resolved by consensus and by consulting a third investigator.

The following data were extracted from each study by two independent investigators: the numbers of true and false positive and negative results, year of publication, information on the index and reference test methods, and information on the study population and country.

We collected 31 studies from international databases (Fig. $1 \mathrm{~A})$ and 15 Korean studies from the international and Korean databases (Fig. 1B). After excluding seven Korean studies from the 31 international ones, we assessed a total of 39 studies (24 foreign and 15 Korean) (Table 2). The details of each study are summarized in Supplemental Data Table S1 [6-44].

\section{Statistical analysis}

For all index tests in all studies, the sensitivity and specificity and their $\mathrm{Cls}$ were calculated from the number of true and false positive and negative results reported in these studies. MetaDiSc version 1.4 (Hospital Universtario Ramon Y Cajal, Madrid, Spain) was used to calculate pooled sensitivities and specificities. We compared and evaluated the foreign and Korean stud- ies to assess differences in the diagnostic performance. Pooled sensitivity and specificity for the three test types in the Korean study group were calculated and compared with those of the foreign studies. Among NAATs, sensitivities and specificities were compared separately for Xpert $C$. difficile, BD Max Cdiff, and AdvanSure CD. Further, automated and non-automated EIAs were compared. In addition, hypothetical PPVs and NPVs were calculated using a prevalence of CDI of $5 \%, 10 \%, 20 \%$, and $50 \%$ in the population tested according to the predictive value theory (Bayes theorem) [45]. The pooled sensitivities and specificities compared with TC in 39 studies searched in international and Korean database were used to compute hypothetical PPVs and NPVs.

Pooled sensitivity and specificity were compared using Fisher's exact test or chi-square test, as appropriate. The data were analyzed using Microsoft Excel 2016 (Microsoft, Redmond, WA, USA). Statistical analysis was performed using MetaDiSc version 1.4 and MedCalc version 10.0 (MedCalc Software Bvba, Ostend, Belgium).

\section{RESULTS}

Pooled sensitivities/specificities of index tests and the sensitivity and specificity of the index test in each study are shown in Table 1 and Supplemental Data Table S2, respectively. GDH EIA and NAAT showed sensitivity and specificity of $90 \%$ or higher, and toxin AB EIA had the highest specificity but low sensitivity (Table 2). The pooled sensitivity of toxin AB EIAs in Korean studies was significantly higher than that in foreign studies. Meanwhile, the pooled specificities of NAATs in foreign studies were significantly higher than those in Korean studies (Table 3). 
Chung HS, et al.

Systematic review of laboratory diagnosis of CDI

A

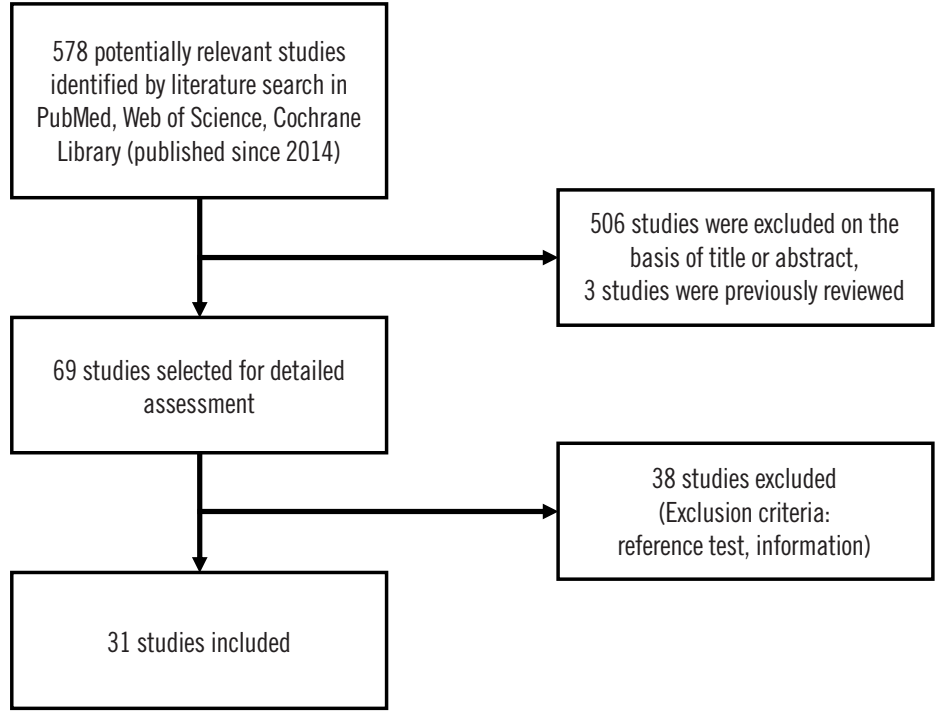

B

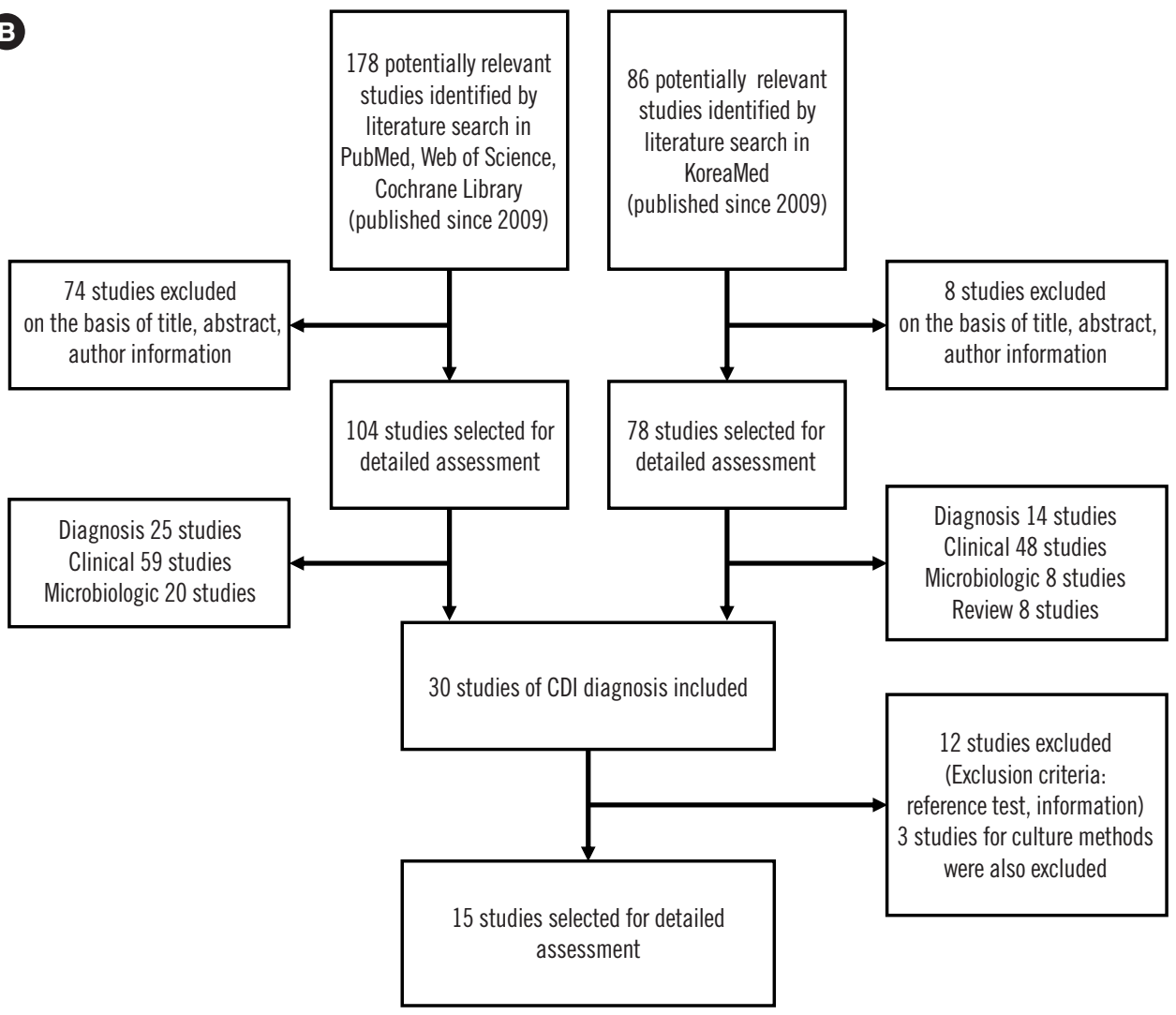

Fig. 1. Summary of the selection process for studies in international databases (A) and Korean studies in international and Korean databases (B).

As the sensitivity of NAAT was significantly lower in foreign studies than that in Korean studies, the methods commonly used in Korea were analyzed separately. The pooled sensitivity of Xpert $C$. difficile, which is the most commonly used NAAT in Korea, was significantly higher than that of all NAATs combined
$(P=0.01)$, and no statistically significant difference in sensitivity was found for BD MAX Cdiff and AdvanSure CD $(P=0.89$ and 0.78 , respectively). The pooled specificities of BD MAX Cdiff and AdvanSure CD were significantly higher than that of all NAATs combined ( $P=0.01$ and 0.05 , respectively). 


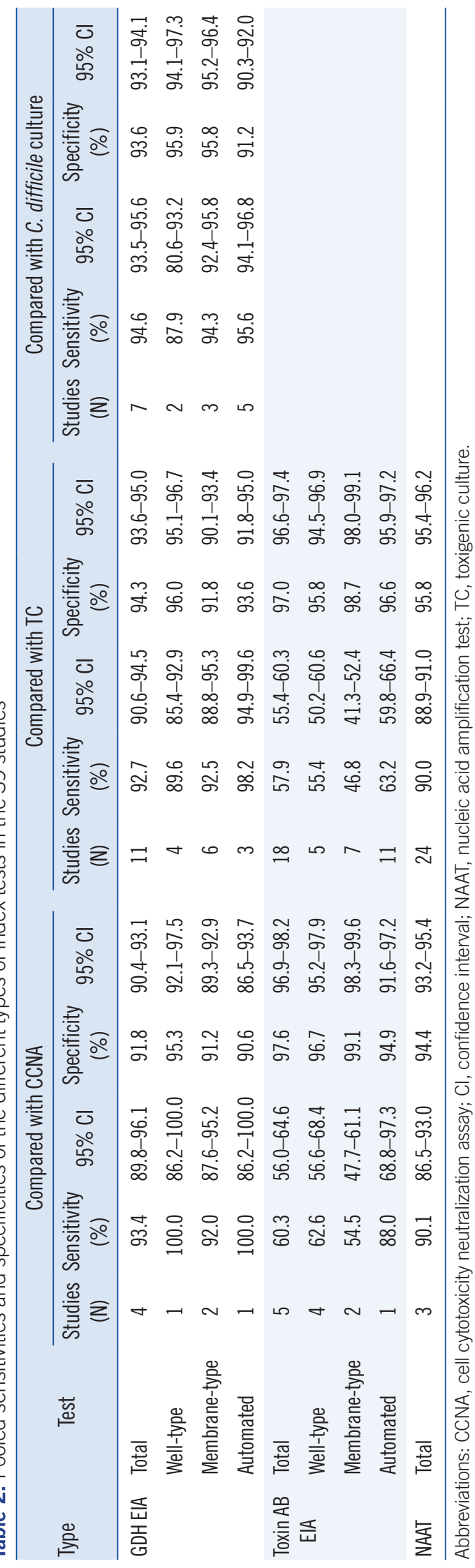

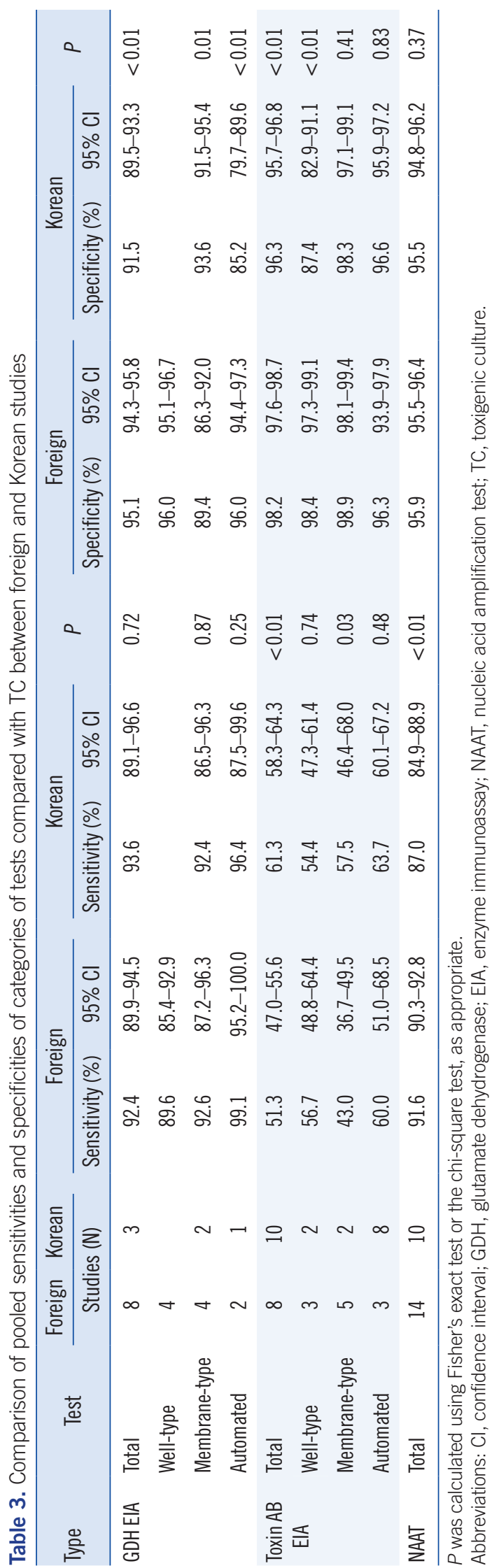


Table 4. Comparison of pooled sensitivities and specificities of the different types of index tests compared with TC between automated and non-automated methods

\begin{tabular}{llccccccc}
\hline Type & \multicolumn{1}{c}{ Test } & Studies (N) & Sensitivity (\%) & $95 \% \mathrm{Cl}$ & $P$ & Specificity (\%) & $95 \% \mathrm{Cl}$ & $P$ \\
\hline GDH EIA & Automated & 3 & 98.2 & $94.9-99.6$ & $<0.01$ & 93.6 & $91.8-95.0$ & 0.23 \\
& Non-automated & 10 & 91.1 & $88.4-93.3$ & & 94.6 & $93.7-95.3$ \\
\multirow{2}{*}{ Toxin AB ElA } & Automated & 11 & 63.2 & $59.8-66.4$ & $<0.01$ & 96.6 & $95.9-97.2$ & 0.03 \\
& Non-automated & 12 & 51.4 & $47.6-55.1$ & & 97.5 & $96.9-98.0$
\end{tabular}

$P$ was calculated using by Fisher's exact test or the chi-square test, as appropriate.

Abbreviations: TC, toxigenic culture; GDH, glutamate dehydrogenase; ElA, enzyme immunoassay.

Table 5. Hypothetical PPV and NPV for different types of index tests in 39 studies at CDI prevalence of 5\%, 10\%, 20\%, and 50\%

\begin{tabular}{|c|c|c|c|c|c|c|c|c|c|}
\hline \multirow{3}{*}{ Type } & \multirow{3}{*}{ Test } & \multicolumn{8}{|c|}{ CDI prevalence } \\
\hline & & \multicolumn{2}{|c|}{$5 \%$} & \multicolumn{2}{|c|}{$10 \%$} & \multicolumn{2}{|c|}{$20 \%$} & \multicolumn{2}{|c|}{$50 \%$} \\
\hline & & PPV & NPV & PPV & NPV & PPV & NPV & PPV & NPV \\
\hline \multirow[t]{4}{*}{ GDH EIA } & Total & 47.5 & 99.6 & 65.6 & 99.1 & 81.1 & 98.1 & 94.5 & 92.8 \\
\hline & Well-type & 54.1 & 99.4 & 71.3 & 98.8 & 84.8 & 97.4 & 95.7 & 90.2 \\
\hline & Membrane-type & 37.3 & 99.6 & 55.6 & 99.1 & 73.8 & 98.0 & 91.9 & 92.4 \\
\hline & Automated & 44.7 & 99.9 & 63.0 & 99.8 & 79.3 & 99.5 & 93.9 & 98.1 \\
\hline \multirow[t]{4}{*}{ Toxin AB EIA } & Total & 50.4 & 97.8 & 68.2 & 95.4 & 82.8 & 90.2 & 95.1 & 69.7 \\
\hline & Well-type & 41.0 & 97.6 & 59.4 & 95.1 & 76.7 & 89.6 & 93.0 & 68.2 \\
\hline & Membrane-type & 65.5 & 97.2 & 80.0 & 94.3 & 90.0 & 88.1 & 97.3 & 65.0 \\
\hline & Automated & 49.5 & 98.0 & 67.4 & 95.9 & 82.3 & 91.3 & 94.9 & 72.4 \\
\hline NAAT & Total & 53.0 & 99.5 & 70.4 & 98.9 & 84.3 & 97.5 & 95.5 & 90.5 \\
\hline
\end{tabular}

Abbreviations: CDI, Clostridioides difficile infection; GDH, glutamate dehydrogenase; EIA, enzyme immunoassay; NAAT, nucleic acid amplification test; PPV, positive predictive value; NPV, negative predictive value.

LIAISON C. difficile GDH/Toxins A\&B and VIDAS C. difficile $\mathrm{GDH} /$ toxin $\mathrm{A} \& B$ were included in automated methods, and welltype and membrane-type ElAs served as non-automated methods. Automated methods had a significantly higher sensitivity than the non-automated methods (Table 4).

Regarding hypothetical PPVs and NPVs of the categories of index tests at different CDI prevalences, PPVs were low at low $\mathrm{CDI}$ prevalence and increased as CDI prevalence increased. In contrast, NPVs were excellent when the CDI prevalence was low and decreased as the prevalence increased. Comparison of PPVs and NPVs of assay types revealed no considerable difference among GDH EIAs, toxin AB EIAs, and NAATs. Among the toxin $A B$ EIAs, the membrane-type toxin $A B$ EIAs had relatively higher PPVs at CDI prevalence of 5\%-20\% than well-type and automated toxin $A B$ EIAs. NPVs of GDH EIAs, toxin AB EIAs, and NAATs did not differ when the CDI prevalence was $10 \%-$ $20 \%$. However, at a CDI prevalence of $50 \%$, NPVs of all types of toxin $A B$ EIAs were relatively low (Table 5).

\section{DISCUSSION}

The analysis of 39 studies available in international and Korean databases revealed that the pooled sensitivities were 92.7\%, $57.9 \%$, and $90.0 \%$ for GDH EIAs, toxin AB EIAs, and NAATs, respectively (Table 2). The pooled sensitivities of GDH EIAs and NAATs were somewhat lower than those reported by the ESCMID group (96\% and 95\%, respectively) [3]. However, there was no difference between the pooled sensitivities of toxin $A B$ EIAs in our study and that by the ESCMID group (57\%) [5]. The pooled specificities were 94.6\%, 97.0\%, and 95.8\% for GDH EIAs, toxin AB EIAs, and NAATs, respectively (Table 2), which were similar to those reported by the ESCMID group (96\%, 99\%, 98\%, respectively) [3].

Our meta-analysis revealed that GDH EIAs had higher sensitivities and narrower sensitivity ranges than toxin $A B$ EIAs. Toxin $A B$ EIAs had both low sensitivities and the widest sensitivity range. However, toxin $A B$ EIAs were the most specific tests. NAATs had a stable sensitivity of approximately $90 \%$ and the 
narrowest sensitivity ranges (Supplemental Data Table S2).

In the 2016 study on CDI by ESCMID [3], the sensitivities of toxin $A B$ ElAs were improved when compared with those reported in 2009 [46]. It is assumed that the main reason for this improvement is that toxin $A$ EIAs were replaced with toxin $A B$ EIAs during 2008-2009. In the present study, no particular improvement was observed, although approximately four years had passed since the last ESCMID study. Despite product development and yearly technological advances, the sensitivity of toxin $A B$ EIAs remains unsatisfactory.

We found that the pooled sensitivity of toxin $A B$ EIAs in Korean studies was significantly higher than that in foreign studies. Among three test methods of toxin AB EIAs, membrane-type methods showed most significant $P$ value when compared with well-type and automated methods. However, this might be explained by the higher proportion of automated EIAs in Korean studies (8/10) than in foreign studies (3/8). The pooled specificities of GDH EIAs and toxin AB EIAs in foreign studies were significantly higher than those in Korean studies (Table 3). Although the specificity of NAATs did not differ significantly between foreign and Korean studies, their sensitivity was significantly lower in Korean studies. However, the pooled sensitivity of Xpert $C$. difficile, which is the most common NAAT in Korea, was significantly higher than that of all NAATs, and no statistically significant difference in sensitivity was found for BD MAX Cdiff and AdvanSure CD.

TC and CCNA, which were used as reference tests, have different targets. TC detects the presence of toxigenic $C$. difficile strains, whereas CCNA detects in-vivo toxin production [2]. The sensitivity and specificity of an index test may differ depending on which reference test was used. This means that toxin $A B$ ElAs may be less sensitive when compared with TC than when compared with CCNA. As NAATs cannot differentiate the presence of in-vivo toxin from in-vitro toxin, they will be less specific when evaluated using CCNA as the reference test. As the CCNA test method is difficult to standardize and maintain, TC was used as the reference test in most foreign studies and all Korean studies.

We found that the pooled sensitivities of automated EIAs were significantly higher than those of non-automated ElAs (well-type and membrane-type) for both GDH and toxin A and B. It is believed that automated tests are less prone to errors than nonautomated tests and therefore have higher sensitivity.

Hypothetical PPV and NPV analysis revealed that PPVs were low at low CDI prevalence and increased as CDI prevalence increased. This result is in line with the findings reported in the
2016 ESCMID study (prevalence 5\%, PPV 34\%-81\%; prevalence $50 \%$, PPV $81 \%-99 \%$ ) [3]. At a CDI prevalence of $5 \%-$ $10 \%$, PPVs of toxin AB EIAs were $69 \%-90 \%$, which were higher than those found in the present study, whereas PPVs of GDH EIAs and NAATs were $34 \%-54 \%$ and $46 \%-64 \%$, respectively, which were lower than those found in the present study. In contrast, NPVs were 95\%-100\% for most tests, but decreased to $80 \%$ for toxin $A B$ EIAs at a CDI prevalence of $50 \%$, which is higher than the NPVs of toxin AB EIAs in the present study. According to the hypothetical PPVs and NPVs in our meta-analysis, in an epidemic outbreak with a CDI prevalence of $50 \%, \mathrm{GDH}$ EIAs and NAATs would have PPVs $\geq 95 \%$ and NPVs $\geq 90 \%$, and toxin ElAs, which have low sensitivity, would have PPVs $\geq 95 \%$ and NPVs around $70 \%$. PPVs of NAATs were the highest, followed by toxin AB EIAs, and GDH EIAs, whereas NPVs remained $\geq 90 \%$ for all test methods except membranetype toxin AB EIAs at CDI prevalence $<20 \%$.

There are several limitations in this study. Although 39 studies were reviewed, some test types or index tests were analyzed with a small number of studies. Additionally, some results of the analysis showed inconsistency. Furthermore, as our study was restricted to studies published in English or Korean, there may be language bias.

The data from this study may be useful for CDI diagnosis in clinical microbiology laboratories and for clinicians diagnosing and treating CDI. Furthermore, this study provided basic data for establishing a standard CDI diagnosis guideline, which will greatly help in the development of national guidelines in Korea.

\section{ACKNOWLEDGEMENTS}

None.

\section{AUTHOR CONTRIBUTIONS}

HSC, JSP, and BMS designed the study and analyzed the data. HSC and BMS wrote the draft. All authors reviewed and approved the manuscript.

\section{CONFLICTS OF INTEREST}

None declared.

\section{RESEARCH FUNDING}

This work was supported by the Research Program funded by the 
Korea Centers for Disease Control and Prevention (2017NP280600).

\section{ORCID}

\author{
Hae-Sun Chung https://orcid.org/0000-0001-6382-4099 \\ Jeong Su Park https://orcid.org/0000-0001-5149-1362 \\ Bo-Moon Shin https://orcid.org/0000-0001-8432-9556
}

\section{REFERENCES}

1. Leffler DA and Lamont JT. Clostridium difficile infection. N Engl J Med 2015;372:1539-48.

2. Bagdasarian N, Rao K, Malani PN. Diagnosis and treatment of Clostridium difficile in adults: a systematic review. JAMA 2015;313:398-408.

3. Crobach MJ, Planche T, Eckert C, Barbut F, Terveer EM, Dekkers OM, et al. European Society of Clinical Microbiology and Infectious Diseases: update of the diagnostic guidance document for Clostridium difficile infection. Clin Microbiol Infect 2016;22(S4):S63-81.

4. McDonald LC, Gerding DN, Johnson S, Bakken JS, Carroll KC, Coffin $\mathrm{SE}$, et al. Clinical practice guidelines for Clostridium difficile infection in adults and children: 2017 update by the Infectious Diseases Society of America (IDSA) and Society for Healthcare Epidemiology of America (SHEA). Clin Infect Dis 2018;66:987-94.

5. Chung HS, Park JS, Shin BM. Laboratory diagnosis of Clostridium difficile infection in Korea: the first national survey. Ann Lab Med 2019; 39:317-21.

6. Alcalá L, Reigadas E, Marin M, Fernández-Chico A, Catalan P, Bouza E. Comparison of GenomEra $C$. difficile and Xpert $C$. difficile as confirmatory tests in a multistep algorithm for diagnosis of Clostridium difficile infection. J Clin Microbiol 2015;53:332-5.

7. Banz A, Lantz A, Riou B, Foussadier A, Miller M, Davies K, et al. Sensitivity of single-molecule array assays for detection of Clostridium difficile toxins in comparison to conventional laboratory testing algorithms. J Clin Microbiol 2018;56:e00452-18.

8. Beck ET, Buchan BW, Riebe KM, Alkins BR, Pancholi P, Granato PA, et al. Multicenter evaluation of the Quidel Lyra Direct $C$. difficile nucleic acid amplification assay. J Clin Microbiol 2014;52:1998-2002.

9. Blaich A, Frei R, Castellano C, Kiessling C, Geschke A, Rentsch KM, et al. Evaluation of two novel chemiluminescence immunoassays for the detection of Clostridium difficile glutamate dehydrogenase and toxin A\&B. J Microbiol Methods 2017;135:63-5.

10. Carson KC and Riley TV. Comparison of the Vidas C. difficile and Quik Chek-60 glutamate dehydrogenase assays for the detection of Clostridium difficile in faecal samples. Pathology 2016;48:506-8.

11. Cheng JW, Xiao M, Kudinha T, Xu ZP, Sun LY, Hou X, et al. The role of glutamate dehydrogenase (GDH) testing assay in the diagnosis of Clostridium difficile infections: a high sensitive screening test and an essential step in the proposed laboratory diagnosis workflow for developing countries like China. PLoS One 2015;10:e0144604.

12. Davies KA, Berry CE, Morris KA, Smith R, Young S, Davis TE, et al. Comparison of the Vidas $C$. difficile $\mathrm{GDH}$ automated enzyme-linked fluorescence immunoassay (ELFA) with another commercial enzyme immunoassay (EIA) (Quik Chek-60), two selective media, and a PCR assay for gluD for detection of Clostridium difficile in fecal samples. J Clin Microbiol 2015;53:1931-4.

13. Gomez EJ, Montgomery S, Alby K, Robinson DP, Roundtree SS, Blecker-Shelly D, et al. Poor yield of Clostridium difficile testing algorithms us- ing glutamate dehydrogenase antigen and $C$. difficile toxin enzyme immunoassays in a pediatric population with declining prevalence of clostridium difficile strain BI/NAP1/027. Diagn Microbiol Infect Dis 2018; 91:229-32.

14. Hong G, Park KS, Ki CS, Lee NY. Evaluation of the illumigene $C$. difficile assay for toxigenic Clostridium difficile detection: a prospective study of 302 consecutive clinical fecal samples. Diagn Microbiol Infect Dis 2014;80:177-80.

15. Jazmati N, Wiegel P, Ličanin B, Plum G. Evaluation of the Qiagen artus $C$. difficile QS-RGQ kit for detection of Clostridium difficile toxins $A$ and $B$ in clinical stool specimens. J Clin Microbiol 2015;53:1942-4.

16. Jensen MB, Olsen KE, Nielsen XC, Hoegh AM, Dessau RB, Atlung T, et al. Diagnosis of Clostridium difficile: real-time PCR detection of toxin genes in faecal samples is more sensitive compared to toxigenic culture. Eur J Clin Microbiol Infect Dis 2015;34:727-36.

17. Johansson K, Karlsson H, Norén T. Clostridium difficile infection diagnostics - evaluation of the C. DIFF Quik Chek Complete assay, a rapid enzyme immunoassay for detection of toxigenic $C$. difficile in clinical stool samples. APMIS 2016;124:1016-20.

18. Kilic A, Alam MJ, Tisdel NL, Shah DN, Yapar M, Lasco TM, et al. Multiplex real-time PCR method for simultaneous identification and toxigenic type characterization of Clostridium difficile from stool samples. Ann Lab Med 2015;35:306-13.

19. Kim H, Kim WH, Kim M, Jeong SH, Lee K. Evaluation of a rapid membrane enzyme immunoassay for the simultaneous detection of glutamate dehydrogenase and toxin for the diagnosis of Clostridium difficile infection. Ann Lab Med 2014;34:235-9.

20. Kim H, Jeong SH, Kim M, Lee Y, Lee K. Detection of Clostridium difficile toxin A/B genes by multiplex real-time PCR for the diagnosis of $C$. difficile infection. J Med Microbiol 2012;61:274-7.

21. Kosai K, Iwanaga Y, Akamatsu N, Okada Y, Kaku N, Uno N, et al. Performance evaluation of the Verigene ${ }^{\circledR}$ Clostridium difficile nucleic acid test, an automated multiplex molecular testing system for detection of $C$. difficile toxin. J Infect Chemother 2017;23:674-7.

22. Legaria MC, Rollet R, Di Martino A, Castello L, Barberis C, Rossetti MA, et al. Detection of toxigenic Clostridioides [Clostridium] difficile: Usefulness of two commercially available enzyme immunoassays and a PCR assay on stool samples and stool isolates. Rev Argent Microbiol 2018; 50:36-44.

23. Makristathis A, Zeller I, Mitteregger D, Kundi M, Hirschl AM. Comprehensive evaluation of chemiluminescent immunoassays for the laboratory diagnosis of Clostridium difficile infection. Eur J Clin Microbiol Infect Dis 2017;36:1253-9.

24. Moon HW, Kim HN, Kim JY, Hur M, Kim H, Yun YM. Performance of the artus C. difficile QS-RGQ Kit for the detection of toxigenic Clostridium difficile. Clin Biochem 2017;50:84-7.

25. Moon HW, Kim HN, Hur M, Shim HS, Kim H, Yun YM. Comparison of diagnostic algorithms for detecting toxigenic Clostridium difficile in routine practice at a tertiary referral hospital in Korea. PLoS One 2016; 11:e0161139.

26. Morinaga Y, Akamatsu N, Matsuda J, Tateno H, Tomaru T, Tanaka A, et al. Diagnostic utilities of a fully automated molecular test for toxigenic Clostridium difficile. J Infect Chemother 2018;24:88-91.

27. Neuendorf M, Guadarrama-Gonzalez R, Lamik B, MacKenzie CR. A prospective study of two isothermal amplification assays compared with real-time PCR, CCNA and toxigenic culture for the diagnosis of Clostridium difficile infection. BMC Microbiol 2016;16:19.

28. Olling A, Leidinger $\mathrm{H}$, Hoffmann R. Comparison of enzyme immunoassays and rapid diagnostic tests for Clostridium difficile glutamate dehydrogenase and toxin $\mathrm{a}+\mathrm{B}$ to toxinogenic culture on a highly selective 
chromogenic medium. Eur J Clin Microbiol Infect Dis 2016;35:1655-9.

29. Paitan Y, Miller-Roll T, Adler A. Comparative performance study of six commercial molecular assays for rapid detection of toxigenic Clostridium difficile. Clin Microbiol Infect 2017;23:567-72.

30. Peterson LR, Young SA, Davis TE, Jr., Wang ZX, Duncan J, Noutsios C, et al. Evaluation of the cobas Cdiff test for detection of toxigenic Clostridium difficile in stool samples. J Clin Microbiol 2017;55:3426-36.

31. Putsathit P, Morgan J, Bradford D, Engelhardt N, Riley TV. Evaluation of the BD Max Cdiff assay for the detection of toxigenic Clostridium difficile in human stool specimens. Pathology 2015;47:165-8.

32. Rajabally N, Kullin B, Ebrahim K, Brock T, Weintraub A, Whitelaw A, et al. A comparison of Clostridium difficile diagnostic methods for identification of local strains in a South African centre. J Med Microbiol 2016; 65:320-7.

33. Seo JY, Jeong JH, Kim KH, Ahn JY, Park PW, Seo YH. Laboratory diagnosis of Clostridium difficile infection: Comparison of Techlab C. diff Quik Chek Complete, Xpert C. difficile, and multistep algorithmic approach. J Clin Lab Anal 2017;31:e22135.

34. Shin BM, Lee EJ, Moon JW, Lee SY. Evaluation of the VIDAS glutamate dehydrogenase assay for the detection of Clostridium difficile. Anaerobe 2016;40:68-72.

35. Shin BM, Yoo SM, Shin WC. Evaluation of Xpert C. difficile, BD MAX Cdiff, IMDx C. difficile for Abbott m2000, and Illumigene C. difficile assays for direct detection of toxigenic Clostridium difficile in stool specimens. Ann Lab Med 2016;36:131-7.

36. Shin BM, Mun SJ, Yoo SJ, Kuak EY. Comparison of BD GeneOhm Cdiff and Seegene Seeplex ACE PCR assays using toxigenic Clostridium difficile culture for direct detection of $t c d B$ from stool specimens. J Clin Microbiol 2012;50:3765-7.

37. Shin S, Kim M, Kim M, Lim H, Kim H, Lee K, et al. Evaluation of the Xpert Clostridium difficile assay for the diagnosis of Clostridium difficile infection. Ann Lab Med 2012;32:355-8.
38. Shin BM, Lee EJ, Kuak EY, Yoo SJ. Comparison of VIDAS CDAB and CDA immunoassay for the detection of Clostridium difficile in a tcd $A^{-}$ tcd $B^{+}$C. difficile prevalent area. Anaerobe 2009;15:266-9.

39. Shin BM, Kuak EY, Lee EJ, Songer JG. Algorithm combining toxin immunoassay and stool culture for diagnosis of Clostridium difficile infection. J Clin Microbiol 2009;47:2952-6.

40. Shin BM, Yoo SJ, Oh HJ. Comparison of two enzyme immunoassay for detection of Clostridium difficile toxin A and toxin B. Korean J Lab Med 2009;29:122-6.

41. Silva RO, Vilela EG, Neves MS, Lobato FC. Evaluation of three enzyme immunoassays and a nucleic acid amplification test for the diagnosis of Clostridium difficile-associated diarrhea at a university hospital in Brazil. Rev Soc Bras Med Trop 2014;47:447-50.

42. Soh YS, Yang JJ, You E, La Jeon Y, Kim MJ, Nam YS, et al. Comparison of two molecular methods for detecting toxigenic Clostridium difficile. Ann Clin Lab Sci 2014;44:27-31.

43. Tojo M, Nagamatsu M, Hayakawa K, Mezaki K, Kirikae T, Ohmagari N. Evaluation of an automated rapid diagnostic test for detection of Clostridium difficile. PLoS One 2014;9:e106102.

44. Yoo J, Lee H, Park KG, Lee GD, Park YG, Park YJ. Evaluation of 3 automated real-time PCR (Xpert $C$. difficile assay, BD MAX Cdiff, and IMDX C. difficile for Abbott m2000 assay) for detecting Clostridium difficile toxin gene compared to toxigenic culture in stool specimens. Diagn Microbiol Infect Dis 2015;83:7-10.

45. Jhang JS and Lifshitz MS. Postanalysis: Medical Decision Making. In: Mcpherson RA and Pincus MR, eds. Henry's clinical diagnosis and management by laboratory methods. 23rd ed. Philadelphia: Elsevier, Saunders, 2016:73-83.

46. Crobach MJ, Dekkers OM, Wilcox MH, Kuijper EJ. European Society of Clinical Microbiology and Infectious Diseases (ESCMID): data review and recommendations for diagnosing Clostridium difficile-infection (CDI). Clin Microbiol Infect 2009;15:1053-66. 
Chung HS, et al.

Systematic review of laboratory diagnosis of CDI

Supplemental Data Table S1. Characteristics of the studies included

\begin{tabular}{|c|c|c|c|c|c|}
\hline Reference & Country & $\mathrm{N}$ & Positive rate (\%) & Reference test & Index test \\
\hline \multirow[t]{3}{*}{ [6] } & Spain & 979 & 12.0 & CCNA & C. Diff Quik Chek Complete \\
\hline & & 103 & 47.6 & & Xpert C. difficile \\
\hline & & & & & GenomEra C. difficile \\
\hline [7] & Canada, France & 240 & 27.5 & CCNA & C. difficile Tox A/B ॥ \\
\hline \multirow[t]{2}{*}[8]{} & USA & 601 & 13.3 & CCNA & Lyra Direct $C$. difficile \\
\hline & & 601 & 18.6 & TC & \\
\hline \multirow[t]{3}{*}{ [9] } & Switzerland & 545 & 14.3 & TC & C. Diff Chek-60 \\
\hline & & & & & LIAISON C. difficile GDH \\
\hline & & & & & LIAISON C. difficile Toxins A\&B \\
\hline \multirow[t]{2}{*}[10]{} & Australia & 403 & 11.9 & C. difficile culture & VIDAS C. difficile GDH \\
\hline & & & & & C. Diff Quik Chek-60 GDH \\
\hline$[11]$ & China & 416 & 26.9 & C. difficile culture & VIDAS C. difficile GDH \\
\hline \multirow[t]{3}{*}[12]{} & UK, USA & 1,914 & & C. difficile culture & C. Diff Quik Chek-60 \\
\hline & & & 18.7 & ChromID & VIDAS C. difficile GDH \\
\hline & & & 16.4 & CCFA & \\
\hline \multirow[t]{6}{*}[13]{} & USA & 211 & 15.6 & TC & C. Diff Quik Chek Complete \\
\hline & & & & & AmpliVue C. difficile \\
\hline & & & & & BD MAX Cdiff \\
\hline & & & & & Illumigene $C$. difficile \\
\hline & & & & & Lyra Direct C. difficile \\
\hline & & & & & Xpert C. difficile /Epi \\
\hline \multirow[t]{2}{*}[14]{} & Korea & 302 & 19.5 & TC & VIDAS C. difficile toxin A\&B \\
\hline & & & & & Illumigene $C$. difficile \\
\hline \multirow[t]{2}{*}{ [15] } & Germany & 199 & 14.1 & TC & artus C. difficile QS-RGQ \\
\hline & & & & & Xpert $C$. difficile \\
\hline \multirow[t]{3}{*}[16]{} & Denmark & 299 & 12.7 & TC & Illumigene $C$. difficile \\
\hline & & & & & PCRFast C. difficile \\
\hline & & & & & Xpert C. difficile \\
\hline$[17]$ & Sweden & 419 & 16.2 & C. difficile culture & C. Diff Quik Chek Complete \\
\hline$[18]$ & USA & 351 & 13.1 & TC & BD GeneOhm Cdiff \\
\hline \multirow[t]{2}{*}{ [19] } & Korea & 608 & 9.0 & TC & C. Diff Quik Chek Complete \\
\hline & & & & & VIDAS C. difficile toxin A\&B \\
\hline \multirow[t]{2}{*}[20]{} & Korea & 127 & 8.7 & TC & VIDAS C. difficile toxin A\&B \\
\hline & & & & & AdvanSure CD \\
\hline \multirow[t]{3}{*}[21]{} & Japan & 118 & 28.0 & TC & C. Diff Quik Chek Complete \\
\hline & & & & & X/Pect Toxin A/B \\
\hline & & & & & Verigene CDF \\
\hline \multirow[t]{2}{*}{ [22] } & Argentina & 250 & 42.8 & CCNA & C. Diff Quik Chek Complete \\
\hline & & & & & RIDASCREEN C. difficile Toxin A/B \\
\hline
\end{tabular}


Supplemental Data Table S1. Continued

\begin{tabular}{|c|c|c|c|c|c|}
\hline Reference & Country & $\mathrm{N}$ & Positive rate (\%) & Reference test & Index test \\
\hline \multirow[t]{5}{*}[23]{} & Austria & 300 & 14.0 & C. difficile culture & C. diff Chek-60 \\
\hline & & & 11.7 & TC & C. difficile Tox A/B II \\
\hline & & & 8.3 & CCNA & LIAISON C. difficile GDH \\
\hline & & & & & LIAISON C. difficile Toxins A\&B \\
\hline & & & & & Xpert C. difficile \\
\hline \multirow[t]{2}{*}{ [24] } & Korea & 258 & 21.3 & TC & artus C. difficile QS-RGQ \\
\hline & & & & & Xpert C. difficile \\
\hline \multirow[t]{3}{*}{ [25] } & Korea & 271 & 20.3 & TC & VIDAS C. difficile GDH \\
\hline & & & & & VIDAS C. difficile toxin A\&B \\
\hline & & & & & Xpert C. difficile \\
\hline \multirow[t]{2}{*}{ [26] } & Japan & 231 & 22.9 & TC & BD MAX Cdiff \\
\hline & & & & & C. Diff Quik Chek Complete \\
\hline \multirow[t]{2}{*}[27]{} & Germany & 989 & 16.9 & TC & AmpliVue C. difficile \\
\hline & & & & & Illumigene $C$. difficile \\
\hline \multirow[t]{3}{*}[28]{} & Germany & 500 & 14.8 & C. difficile culture & RIDASCREEN GDH \\
\hline & & & 12.0 & TC & RIDASCREEN C. difficile Toxin A/B \\
\hline & & & & & RIDA QUIK \\
\hline \multirow[t]{6}{*}{ [29] } & Israel & 209 & 46.9 & TC & AmpliVue C. difficile \\
\hline & & & & & BDmax Cdiff \\
\hline & & & & & GenomEra C. difficile \\
\hline & & & & & Illumigene $C$. difficile \\
\hline & & & & & Simplexa C. difficile Universal Direct \\
\hline & & & & & Xpert C. difficile \\
\hline [30] & USA & 682 & 20.7 & TC & Cobas Cdiff \\
\hline \multirow[t]{3}{*}{ [31] } & Australia & & & TC & C. diff Chek-60 \\
\hline & & 334 & 7.2 & Enrichment* & BD Max Cdiff \\
\hline & & 332 & 6.6 & Direct $^{\dagger}$ & \\
\hline \multirow[t]{4}{*}[32]{} & South Africa & 141 & 21.3 & $\mathrm{TC}$ & ImmunoCard Toxins A \& B \\
\hline & & & & & VIDAS C. difficile Toxin A \& B \\
\hline & & & & & GenoType Cdiff \\
\hline & & & & & Xpert C. difficile \\
\hline \multirow[t]{2}{*}{ [33] } & Korea & 191 & 16.8 & TC & C. diff Quik Chek Complete \\
\hline & & & & & Xpert C. difficile \\
\hline [34] & Korea & 350 & 30.9 & C. difficile culture & VIDAS C. difficile GDH \\
\hline \multirow[t]{4}{*}{ [35] } & Korea & 339 & 25.7 & TC & BD MAX Cdiff \\
\hline & & & & & Illumigene $C$. difficile \\
\hline & & & & & IMDx C. difficile \\
\hline & & & & & Xpert C. difficile \\
\hline \multirow[t]{2}{*}{ [36] } & Korea & 243 & 28.8 & $\mathrm{TC}$ & BD Gene0hm Cdiff \\
\hline & & & & & Seeplex Diarrhea-B1 ACE detection \\
\hline
\end{tabular}


Chung HS, et al.

Systematic review of laboratory diagnosis of CDI

Supplemental Data Table S1. Continued

\begin{tabular}{|c|c|c|c|c|c|}
\hline Reference & Country & $\mathrm{N}$ & Positive rate (\%) & Reference test & Index test \\
\hline \multirow[t]{2}{*}[37]{} & Korea & 253 & 19.4 & TC & VIDAS C. difficile toxin A\&B \\
\hline & & & & & Xpert C. difficile \\
\hline [38] & Korea & 555 & 21.8 & TC & VIDAS C. difficile toxin A\&B \\
\hline [39] & Korea & 1,596 & 22.7 & TC & VIDAS C. difficile toxin A\&B \\
\hline \multirow[t]{2}{*}[40]{} & Korea & 228 & 51.3 & TC & C. difficile Tox A/B II \\
\hline & & & & & VIDAS C. difficile toxin A\&B \\
\hline \multirow[t]{4}{*}[41]{} & Brazil & 92 & 27.2 & CCNA & C. difficile Tox A/B II \\
\hline & & & 25.0 & $\mathrm{TC}$ & Remel ProSpecT C. difficile Toxin A/B \\
\hline & & & & & RIDASCREEN C. difficile Toxin A/B \\
\hline & & & & & Simplexa C. difficile Universal Direct \\
\hline \multirow[t]{2}{*}{ [42] } & Korea & 203 & 12.8 & $\mathrm{TC}$ & AdvanSure CD \\
\hline & & & & & Illumigene C. difficile \\
\hline \multirow[t]{2}{*}{ [43] } & Japan & 69 & 43.5 & TC & C. Diff Quik Chek Complete \\
\hline & & & & & Verigene CDF \\
\hline \multirow[t]{4}{*}{ [44] } & Korea & 254 & 34.3 & TC & RIDASCREEN C. difficile Toxin A/B \\
\hline & & & & & BD MAX Cdiff \\
\hline & & & & & IMDx C. difficile \\
\hline & & & & & Xpert $C$. difficile \\
\hline
\end{tabular}

*Enrichment in cooked meat broth supplemented with gentamicin, cefoxitin, and cycloserine was undertaken. Taurocholic acid was added to the enrichment broth as a germinant and, after incubation, broths were alcohol shocked and plated onto ChromID C. difficile agar; ${ }^{\dagger}$ Toxigenic culture was performed by direct plating onto ChromID C. difficile agar.

Abbreviations: CCNA, cell cytotoxicity neutralization assay; CCFA, cycloserine-cefoxitin fructose agar; TC, toxigenic culture. 
Supplemental Data Table S2. Sensitivities and specificities of index tests

\begin{tabular}{|c|c|c|c|c|c|}
\hline Index test & Reference & Sensitivity (\%) & $95 \% \mathrm{Cl}$ & Specificity (\%) & $95 \% \mathrm{Cl}$ \\
\hline \multicolumn{6}{|l|}{ Compared with TC } \\
\hline \multicolumn{6}{|l|}{ Well-type GDH EIA } \\
\hline C. diff Chek-60 & [9] & 93.6 & $88.2-99.0$ & 98.5 & $97.4-99.6$ \\
\hline C. diff Chek-60 & [23] & 94.3 & $80.8-99.1$ & 98.1 & $95.7-99.4$ \\
\hline C. diff Chek-60 & [31] & 87.5 & $67.6-97.2$ & 100.0 & $98.8-100.0$ \\
\hline C. diff Chek-60 & {$[31]$} & 95.5 & 77.1-99.2 & 100.0 & $98.8-100.0$ \\
\hline RIDA QUICK & [28] & 81.7 & $69.6-90.5$ & 91.1 & 88.1-93.6 \\
\hline RIDASCREEN GDH & [28] & 88.3 & $77.4-95.2$ & 91.1 & 88.1-93.6 \\
\hline \multicolumn{6}{|l|}{ Membrane-type GDH EIA } \\
\hline C. Diff Quik Chek Complete & [13] & 87.9 & $71.8-96.5$ & 92.1 & $87.2-95.6$ \\
\hline C. Diff Quik Chek Complete & [19] & 91.0 & $85.4-96.6$ & 92.4 & $90.1-94.7$ \\
\hline C. Diff Quik Chek Complete & [21] & 97.0 & $84.2-99.5$ & 71.8 & $61.0-81.0$ \\
\hline C. Diff Quik Chek Complete & [26] & 92.5 & $81.8-97.9$ & 94.4 & 89.9-97.3 \\
\hline C. Diff Quik Chek Complete & [33] & 96.9 & $83.7-99.5$ & 97.5 & $93.7-99.3$ \\
\hline C. Diff Quik Chek Complete & [43] & 93.3 & 77.9-99.0 & 92.3 & 79.1-98.3 \\
\hline \multicolumn{6}{|l|}{ Automated GDH EIA } \\
\hline LIAISON C. difficile GDH & [9] & 98.7 & $96.2-100.0$ & 97.2 & $95.7-98.7$ \\
\hline LIAISON C. difficile GDH & [23] & 100.0 & $89.9-100.0$ & 94.0 & $90.4-96.5$ \\
\hline VIDAS C. difficile GDH & [25] & 96.4 & $87.5-99.6$ & 85.2 & $79.7-89.6$ \\
\hline \multicolumn{6}{|l|}{ Well-type toxin AB EIA } \\
\hline C. difficile Tox A/B II & [23] & 54.3 & $36.7-71.2$ & 97.7 & $95.1-99.2$ \\
\hline C. difficile Tox A/B II & {$[40]$} & 71.8 & $62.7-79.7$ & 70.3 & $60.9-78.6$ \\
\hline C. difficile Tox A/B II & [41] & 65.2 & $44.9-81.2$ & 98.5 & $91.9-99.7$ \\
\hline Remel ProSpecT C. difficile Toxin A/B & [41] & 60.9 & $40.8-77.8$ & 91.3 & $82.3-96.0$ \\
\hline RIDASCREEN C. difficile Toxin A/B & [28] & 51.7 & $38.4-64.8$ & 100.0 & $99.2-100.0$ \\
\hline RIDASCREEN C. difficile Toxin A/B & [41] & 60.9 & $40.8-77.8$ & 97.1 & $90.0-99.2$ \\
\hline RIDASCREEN C. difficile Toxin A/B & [44] & 31.0 & $21.6-41.9$ & 98.8 & $95.7-98.8$ \\
\hline \multicolumn{6}{|l|}{ Membrane-type toxin AB ElA } \\
\hline C. Diff Quik Chek Complete & [13] & 21.2 & $9.0-38.9$ & 98.9 & $96.0-99.8$ \\
\hline C. Diff Quik Chek Complete & [19] & 63.6 & $50.9-76.3$ & 98.0 & $96.8-99.2$ \\
\hline C. Diff Quik Chek Complete & [21] & 45.5 & $28.1-63.6$ & 94.1 & $86.8-98.1$ \\
\hline C. Diff Quik Chek Complete & [26] & 52.8 & $38.6-66.7$ & 100.0 & $97.9-100.0$ \\
\hline C. Diff Quik Chek Complete & [33] & 46.9 & $29.1-65.3$ & 99.4 & $96.5-99.9$ \\
\hline ImmunoCard Toxins A and B & [32] & 40.0 & $22.7-59.4$ & 99.1 & $95.1-100.0$ \\
\hline RIDA QUICK & [28] & 55.0 & $41.6-67.9$ & 99.1 & $97.7-99.8$ \\
\hline X/Pect Toxin A/B & [21] & 27.3 & $13.3-45.5$ & 100.0 & $95.8-100.0$ \\
\hline \multicolumn{6}{|l|}{ Automated toxin AB EIA } \\
\hline LIAISON C. difficile Toxins A\&B & [9] & 60.0 & $51.6-68.4$ & 92.3 & $85.1-99.6$ \\
\hline LIAISON C. difficile Toxins A\&B & [23] & 68.6 & $50.7-83.1$ & 95.5 & $92.2-97.6$ \\
\hline VIDAS C. difficile toxin A\&B & [14] & 40.4 & $27.0-54.9$ & 98.8 & $96.4-99.7$ \\
\hline VIDAS C. difficile toxin A\&B & [19] & 75.5 & $63.5-87.5$ & 97.4 & $96.1-98.7$ \\
\hline
\end{tabular}


Chung HS, et al.

Systematic review of laboratory diagnosis of CDI

Supplemental Data Table S2. Continued

\begin{tabular}{|c|c|c|c|c|c|}
\hline Index test & Reference & Sensitivity (\%) & $95 \% \mathrm{Cl}$ & Specificity (\%) & $95 \% \mathrm{Cl}$ \\
\hline VIDAS C. difficile toxin A\&B & {$[20]$} & 63.6 & $30.9-88.9$ & 100.0 & $96.8-100.0$ \\
\hline VIDAS C. difficile toxin A\&B & {$[25]$} & 49.1 & $35.4-62.9$ & 98.2 & $95.3-99.5$ \\
\hline VIDAS C. difficile toxin A\&B & {$[32]$} & 50.0 & $31.3-68.7$ & 99.1 & $95.1-100.0$ \\
\hline VIDAS C. difficile toxin A\&B & {$[37]$} & 44.4 & $29.7-60.0$ & 100.0 & $98.2-100.0$ \\
\hline VIDAS C. difficile toxin A\&B & [38] & 69.2 & $59.5-77.7$ & 96.7 & $94.5-98.2$ \\
\hline VIDAS C. difficile toxin A\&B & [39] & 67.7 & $62.1-72.9$ & 96.1 & $94.9-97.1$ \\
\hline VIDAS C. difficile toxin A\&B & {$[40]$} & 68.5 & $59.0-77.0$ & 79.2 & $70.0-86.6$ \\
\hline \multicolumn{6}{|l|}{ NAAT } \\
\hline AdvanSure CD & {$[20]$} & 100.0 & $71.3-100.0$ & 98.3 & 93.9-99.7 \\
\hline AdvanSure CD & {$[42]$} & 84.6 & $65.1-95.6$ & 98.3 & $95.1-99.6$ \\
\hline AmpliVue C. difficile & {$[13]$} & 93.9 & 79.7-99.1 & 98.9 & $96.0-99.8$ \\
\hline AmpliVue C. difficile & [27] & 91.0 & $85.6-94.9$ & 89.1 & $86.7-91.1$ \\
\hline AmpliVue C. difficile & [29] & 92.9 & $85.8-97.1$ & 100.0 & $96.7-100.0$ \\
\hline artus C. difficile QS-RGQ & {$[15]$} & 100.0 & $87.5-100.0$ & 89.5 & 83.9-93.6 \\
\hline artus C. difficile QS-RGQ & [24] & 98.2 & $90.2-99.7$ & 93.6 & $89.3-96.5$ \\
\hline BD Gene0hm Cdiff & {$[18]$} & 97.8 & $87.0-99.8$ & 97.7 & $95.1-98.9$ \\
\hline BD GeneOhm Cdiff & {$[36]$} & 95.7 & $88.0-99.1$ & 96.5 & $92.6-98.7$ \\
\hline BD MAX Cdiff & {$[13]$} & 96.9 & $83.7-99.5$ & 100.0 & $97.9-100.0$ \\
\hline BD MAX Cdiff & [26] & 98.1 & $89.9-100.0$ & 98.9 & $96.0-99.9$ \\
\hline BD MAX Cdiff & [29] & 91.8 & $84.5-96.4$ & 100.0 & $96.7-100.0$ \\
\hline BD Max Cdiff & {$[31]$} & 91.7 & 73.0-99.0 & 99.0 & $97.2-99.8$ \\
\hline BD Max Cdiff & {$[31]$} & 95.5 & $77.2-99.9$ & 99.0 & $97.2-99.8$ \\
\hline BD MAX Cdiff & [35] & 88.5 & $79.9-94.3$ & 89.3 & $84.8-92.8$ \\
\hline BD MAX Cdiff & {$[44]$} & 81.6 & $71.9-89.1$ & 95.8 & $91.6-98.3$ \\
\hline Cobas Cdiff & {$[30]$} & 92.9 & $87.4-96.1$ & 98.7 & $97.4-99.4$ \\
\hline GenomEra C. difficile & [29] & 91.8 & $84.5-96.4$ & 99.1 & $95.1-99.9$ \\
\hline GenoType Cdiff & {$[32]$} & 86.7 & $69.3-96.2$ & 88.3 & $80.8-93.6$ \\
\hline Illumigene $C$. difficile & {$[14]$} & 88.1 & $77.1-95.1$ & 96.7 & $93.6-98.6$ \\
\hline Illumigene C. difficile & {$[16]$} & 94.7 & $82.2-99.2$ & 95.8 & $92.6-97.9$ \\
\hline Illumigene $C$. difficile & [27] & 91.6 & $86.3-95.3$ & 90.9 & $88.7-92.8$ \\
\hline Illumigene $C$. difficile & [29] & 92.9 & $85.8-97.1$ & 100.0 & $96.7-100.0$ \\
\hline Illumigene $C$. difficile & [35] & 80.5 & $70.6-88.2$ & 93.7 & $70.6-88.2$ \\
\hline Illumigene C. difficile & {$[42]$} & 92.3 & $74.8-98.8$ & 99.4 & $96.9-99.9$ \\
\hline IMDx C. difficile & {$[35]$} & 85.1 & $75.8-91.8$ & 94.4 & $90.9-96.9$ \\
\hline IMDx C. difficile & {$[44]$} & 62.1 & $51.0-72.3$ & 99.4 & $96.7-99.9$ \\
\hline Lyra Direct C. difficile & {$[8]$} & 82.1 & $73.5-88.5$ & 96.9 & $94.9-98.2$ \\
\hline Lyra Direct C. difficile & {$[8]$} & 89.3 & $81.7-94.1$ & 98.8 & $97.2-99.5$ \\
\hline Lyra Direct C. difficile & {$[8]$} & 85.7 & $77.5-91.4$ & 99.0 & $97.4-99.6$ \\
\hline Lyra Direct $C$. difficile & {$[13]$} & 87.9 & $71.8-96.5$ & 98.9 & $96.0-99.8$ \\
\hline PCRFast $C$. difficile & {$[16]$} & 76.3 & $59.8-88.5$ & 98.1 & $95.6-99.7$ \\
\hline Seeplex Diarrhea-B1 ACE detection & {$[36]$} & 90.0 & $80.5-95.9$ & 97.1 & $93.4-99.0$ \\
\hline
\end{tabular}


Supplemental Data Table S2. Continued

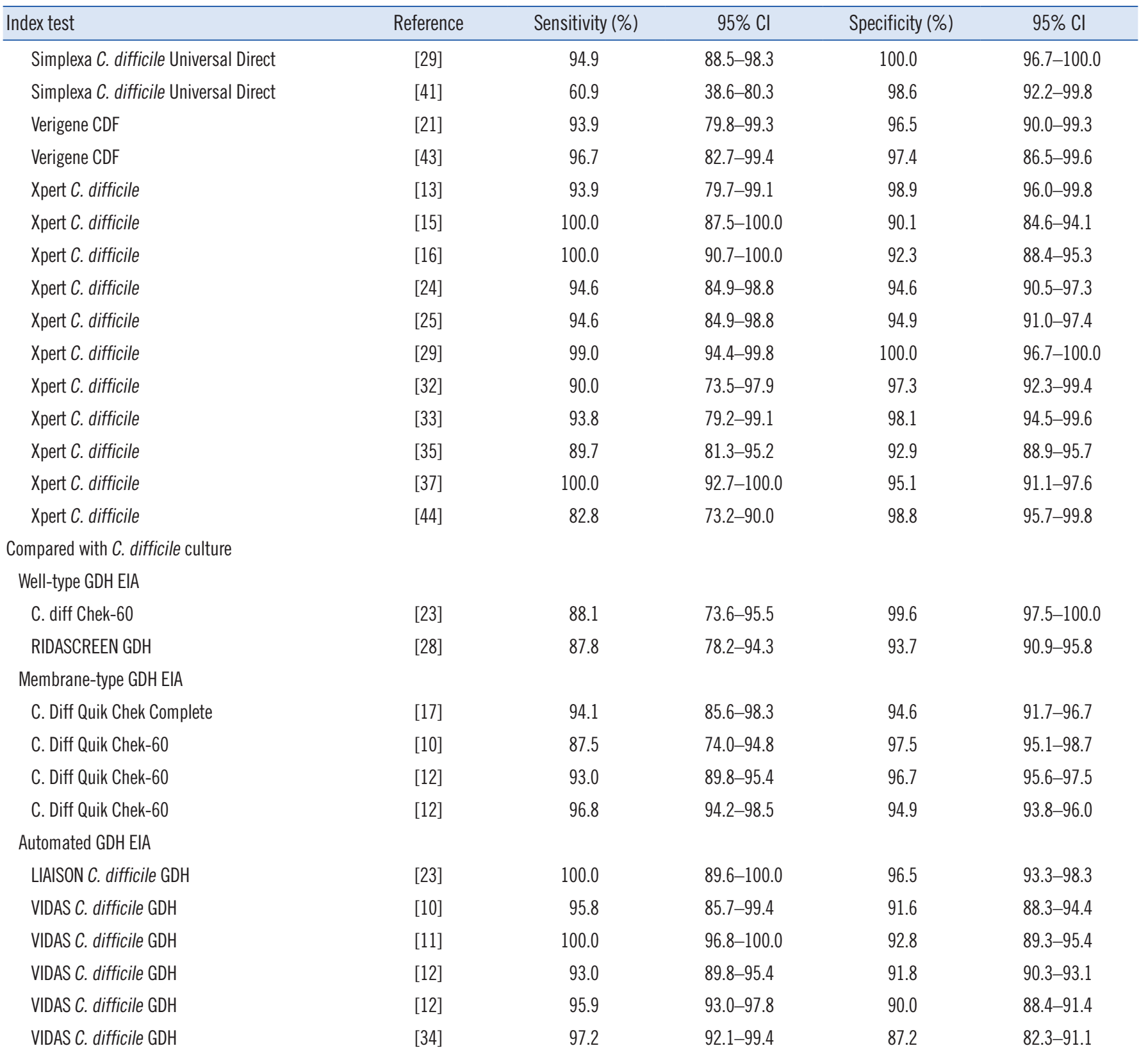

Abbreviations: TC, toxigenic culture; $\mathrm{Cl}$, confidence interval; $\mathrm{GDH}$, glutamate dehydrogenase; EIA, enzyme immunoassay; NAAT, nucleic acid amplification test. 